\title{
DYSLIPIDAEMIA
}

\section{Balancing the benefits and risks of statin therapy}

"The numbers of people who avoid heart attacks and strokes by taking statin therapy are very much larger than the numbers who have side effects with it," claims Rory Collins, lead author of a Review into the efficacy and safety of statin therapy published in The Lancet.

After the first year of therapy, statins reduce the risk of major vascular events by about $25 \%$ for each $1 \mathrm{mmol} / \mathrm{l}$ reduction in the $\mathrm{LDL}$-cholesterol level during each year that the drug continues to be taken. Given that currently available statin regimens can reduce the LDL-cholesterol level by as much as $2 \mathrm{mmol} / \mathrm{l}$, the risk of heart attacks and strokes might be as much as halved in many patients. The absolute benefits of statin therapy depend on an individual's absolute risk of occlusive vascular events (rather than on their baseline blood cholesterol concentration). Accordingly, the 2013
ACC/AHA guidelines emphasize the importance of statin treatment for patients who are at high risk, rather than only those individuals with high cholesterol concentrations. Given that statin therapy continues to reduce the risk of vascular events during each year that it is taken, larger absolute benefits accrue with more prolonged therapy.

Statin therapy is associated with some adverse effects, including myopathy, incident diabetes mellitus, and possibly haemorrhagic stroke. The researchers estimate that treatment of 10,000 patients for 5 years with an effective statin regimen (such as atorvastatin $40 \mathrm{mg}$ daily) would cause five extra cases of myopathy (one of which might progress to rhabdomyolysis), $50-100$ cases of diabetes, and five to ten haemorrhagic strokes. By contrast, the same statin regimen would typically prevent major vascular events from

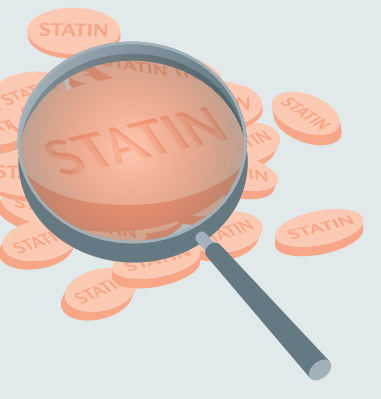

occurring in 1,000 high-risk patients with pre-existing vascular occlusive disease (secondary prevention), and in 500 individuals who are at increased risk but have not previously had a vascular event (primary prevention).

"Cholesterol-lowering therapy is substantially under-used by people at high risk of heart attacks and strokes," highlight the researchers. "Much greater caution is warranted than has sometimes been the case when making claims about possible side effects, since otherwise patients at high risk of heart attacks, strokes, and related deaths, and their doctors, may well be inappropriately dissuaded from using statin therapy despite the proven benefits." Of note, "whereas most of the side effects can be reversed with no residual effects by stopping the statin, the effects of a heart attack or stroke not being prevented are irreversible and can be devastating," concludes Professor Collins.

$$
\text { Gregory B. Lim }
$$
risk of heart attacks and strokes
ORIGINAL ARTICLE Collins, $R$, et al.

Interpretation of the evidence for the efficacy and safety of statin therapy. Lancet http://dx.doi.org/ 10.1016/S0140-6736(16)31357-5 (2016)
Cholesterollowering substantially under-used by people at high 